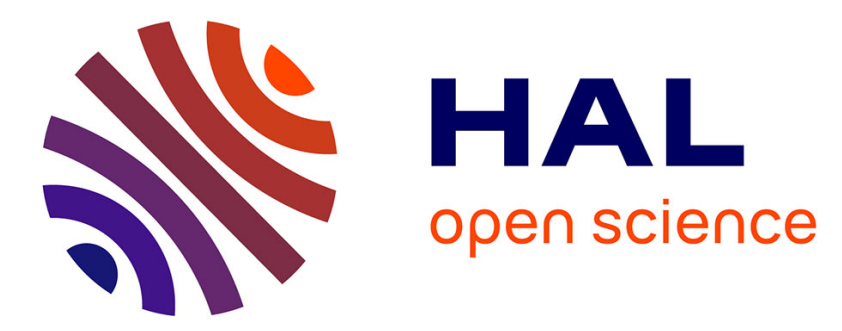

\title{
The component dynamics of miscible binary mixtures of glass-formers: new features
}

Mohamed Shahin Thayyil, Simone Capaccioli, Pierangelo Rolla, Kia Ling Ngai

\section{To cite this version:}

Mohamed Shahin Thayyil, Simone Capaccioli, Pierangelo Rolla, Kia Ling Ngai. The component dynamics of miscible binary mixtures of glass-formers: new features. Philosophical Magazine, 2008, 88 (33-35), pp.4047-4055. 10.1080/14786430802562140 . hal-00513990

\section{HAL Id: hal-00513990 \\ https://hal.science/hal-00513990}

Submitted on 1 Sep 2010

HAL is a multi-disciplinary open access archive for the deposit and dissemination of scientific research documents, whether they are published or not. The documents may come from teaching and research institutions in France or abroad, or from public or private research centers.
L'archive ouverte pluridisciplinaire HAL, est destinée au dépôt et à la diffusion de documents scientifiques de niveau recherche, publiés ou non, émanant des établissements d'enseignement et de recherche français ou étrangers, des laboratoires publics ou privés. 


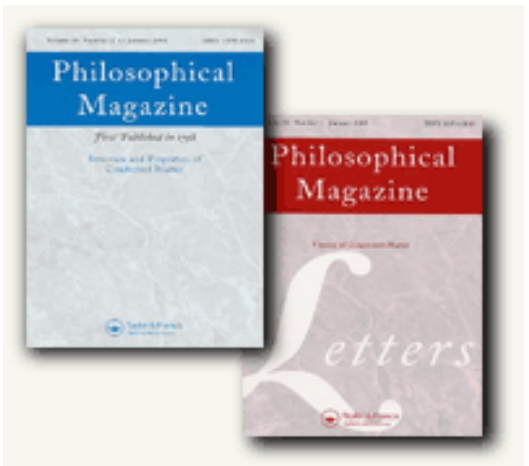

The component dynamics of miscible binary mixtures of glass-formers: new features

\begin{tabular}{|c|c|}
\hline Journal: & Philosophical Magazine \& Philosophical Magazine Letters \\
\hline Manuscript ID: & TPHM-08-May-0182.R1 \\
\hline Journal Selection: & Philosophical Magazine \\
\hline $\begin{array}{r}\text { Date Submitted by the } \\
\text { Author: }\end{array}$ & 15-Sep-2008 \\
\hline Complete List of Authors: & $\begin{array}{l}\text { Thayyil, Mohamed Shahin; University of Pisa, Dipartimento di } \\
\text { Fisica; University of Pisa, Dipartimento di Fisica } \\
\text { Capaccioli, Simone; CNR-INFM, polyLab, \& Physics Department, } \\
\text { Università degli Studi di Pisa, Physics } \\
\text { Rolla, Pierangelo; CNR-INFM, polyLab, \& Physics Department, } \\
\text { Università degli Studi di Pisa, Physics } \\
\text { Ngai, Kia; Naval Research Laboratory, Code } 6807\end{array}$ \\
\hline Keywords: & disordered systems, glass transition \\
\hline Keywords (user supplied): & dielectric relaxation, binary mixures \\
\hline
\end{tabular}

\section{5) ScholaronE \\ Manuscript Central}




\title{
The component dynamics of miscible binary mixtures of glass- formers: new features
}

\author{
MOHAMED SHAHIN THAYYIL $\dagger \neq$, SIMONE CAPACCIOLI* ${ }^{*}$, PIERANGELO ROLLA $\dagger$ and KIA L. \\ NGAI§ \\ † CNR-INFM, polyLab, \& Physics Department, Università degli Studi di Pisa, Largo Pontecorvo 3 , I-56127 \\ Pisa (Italy) \\ ¥ Department of Physics, University of Calicut, Kerala, INDIA \\ $\S$ Naval Research Laboratory, Washington DC 20375-5320 USA \\ *Corresponding author. Email: Simone.Capaccioli@df.unipi.it
}

\begin{abstract}
Opposite effects on the width of the dispersion of the $\alpha$-relaxation and the separation on the time scale of the Johari-Goldstein $\beta$-relaxation from the $\alpha$-relaxation are predicted for guest molecules dissolved in a host depending on whether the glass transition temperature of the host is higher or lower than that of the guest. These predictions are tested by dielectric relaxation measurements on mixtures of two glass-forming mixtures. The results are in agreement with the predictions.
\end{abstract}

Keywords: dielectric relaxation; glass-forming liquids; secondary relaxation; binary mixures

\section{Introduction}

Explanation of the dynamics of a component in binary miscible mixtures found by experimental measurements has occupied the attention of the research community. Of interest is the change of the dynamics of a glass-former (the guest) when it is homogeneously mixed with another glass-former (the host). By now, it is well known that the guest and the host have their own individual dynamics in the mixture [1--6]. The most noticeable and easily observed change of the dynamics of the guest is that of its primary $\alpha$-relaxation, including the shift of the $\alpha$-relaxation time, $\tau_{\alpha, g}$, to either longer or shorter times depending on the composition of the mixture. This is the primary concern of most theoretical models of mixtures [7--9]. However, the focus of these models solely on the $\alpha$-relaxation of a component may have left out an important aspect of the dynamics coming out to light in recent years. The aspect is the possible fundamental role played by secondary relaxation which has properties either mimicking or correlated with that of the $\alpha$-relaxation in neat glass-formers. Some examples of experimental data showing the strong connection of the $\alpha$-relaxation with secondary relaxation belonging to this special class, called Johari-Goldstein (JG) $\beta$-relaxation, are given in Refs.[10-14]. The connections imply that the $\alpha$ - and JG $\beta$-relaxations are inseparable processes in considering glass transition. Remarkably, the connection is passed onto the $\alpha$ - and JG $\beta$-relaxations of a component in mixtures of two van der Waals glass-formers. An example is the invariance of the ratio of $\tau_{\alpha g}$ to $\tau_{J G, g}$, the relaxation time of the JG $\beta$-relaxation, to widely different combinations of pressure and temperature that maintain $\tau_{\alpha, g}$ constant [15-16]. This is a remarkable result because in these experiments the specific volume varies over a significant range, and yet $\tau_{\alpha g} / \tau_{J G, g}$ is invariant at constant $\tau_{\alpha g}$. So far, there is only one 
published model of component dynamics in mixtures based on the Coupling Model (CM) that addresses both the $\alpha$ - and JG $\beta$-relaxations of a component, and can explain their various connections [6, 15-17]. In all mixtures considered thus far, the guest in its pure state has a lower glass transition temperature than that of the host. Therefore, in the mixture, replacement of guest molecules by the less mobile host molecules will stretch the $\alpha$-relaxation process associated with the guest molecules to longer times. This heuristic description suggests increased stretching of the correlation function or equivalently broadening of the frequency dispersion of the guest component $\alpha$-relaxation. Also, the less mobile host molecules enhance the intermolecular constraints on the motions of molecules participating in the guest component $\alpha$-relaxation. From this, the CM expects the coupling parameter $n_{g}$ of the guest molecule in the mixture to be larger than its value $n_{0}$ of the guest molecules in the pure state. Since the $\alpha$-correlation functions of the guest in the mixture and in the pure state are given by $\phi(t)=\exp \left\{-\left(t / \tau_{\alpha g}\right)^{1-n_{g}}\right\}$ and $\phi(t)=\exp \left\{-\left(t / \tau_{\alpha}\right)^{1-n_{0}}\right\}$ respectively, from $n_{g}>n_{0}$ we expect the width of the guest molecules $\alpha$-dispersion is increased when mixed with the host having higher $T_{g}$. As shown in Ref.[6] and will not be repeated here, the other prediction of the CM is on the separation between the $\alpha$-relaxation and the JG $\beta$-relaxation. The separation, measured by the difference of the logarithm of their relaxation times, is given by

$\log _{10} \tau_{\alpha}-\log _{10} \tau_{J G}=n_{0}\left(\log \tau_{\alpha}+11.7\right)$

for the pure guest, and

$$
\log _{10} \tau_{\alpha g}-\log _{10} \tau_{J G, g}=n_{g}\left(\log \tau_{\alpha g}+11.7\right)
$$

for the guest in the mixture. Again, because of $n_{g}>n_{0}$, the separation is larger in the mixture if $\tau_{\alpha}=\tau_{\alpha g}$.

Several previous experimental studies $[15,16,18-22]$ and simulations have verified two simultaneous CM predictions in mixtures where the host has a significantly higher $T_{g}$ than the guest. They are:

(i) The frequency dispersion of the guest $\alpha$-relaxation in the mixture is broader than the pure guest (besides the broadening by composition fluctuations in mixtures).

(ii) The JG $\beta$-relaxation is further separated from the $\alpha$-relaxation in the mixture than in the pure guest. The case of polar guest in apolar host is particularly interesting for dielectric spectroscopy, as the contribution to the overall loss spectrum is mainly due to to the dipoles of the guest molecules. Therefore, dielectric spectroscopy is probing selectively the orientational dynamics of the polar molecules. However, attention has not been given to the CM predictions for the reverse situation, i.e. when the apolar host has a comparable or lower $\mathrm{Tg}$ than the dipolar guest. In this opposite case, the more mobile host molecules mitigate the intermolecular constraints of molecular motions in the guest $\alpha$-relaxation, and hence $n_{g}<n_{0}$. From this, we immediate make the two following predictions.

(iii) The frequency dispersion of the guest $\alpha$-relaxation is narrower in the mixture than in the pure guest (not taking into account of the broadening by composition fluctuation in the mixture).

(iv) Since Eqs.(1) and (2) are general, now they predicts that the separation between the guest $\alpha$ relaxation and the JG $\beta$-relaxation will decrease in the mixture.

The purpose of this paper is to critically test these simultaneous predictions by new analysis of previously obtained dielectric relaxation data of $10 \mathrm{wt} . \%$ dipolar benzylacetate (BzAc) with $T_{g}=165.1 \mathrm{~K}$ in apolar host, isopropylbenzene (IPB) with $T_{g}=128.5 \mathrm{~K}$ or $o$-terphenyl (OTP) with $T_{g}=244 \mathrm{~K}$ [23]. The $T_{g}$ of OTP is 80 degrees higher BzAc, while the $T_{g}$ of IPB is slightly lower than BzAc. Test is also made by newly acquired dielectric relaxation data of dipolar bromoethylbenzene $(\mathrm{BrEBz})$ with $T_{g}=135.6 \mathrm{~K}$ in apolar host, ethylbenzene (EB) with $T_{g}=115 \mathrm{~K}$ or tri-styrene $(3 \mathrm{~S})$ with $T_{g}=232 \mathrm{~K}$. 


\section{Experimental Section}

The details for measurements on BzAc and mixtures with IPB and OTP can be found in Ref.[23--24]. Bromo2-ethylbenzene (BrEBz), ethylbenzene (EB) were obtained from Aldrich and used as received. Tristyrene (3S) with molecular weight $=370 \mathrm{~g} \mathrm{~mol}^{-1}, \mathrm{M}_{\mathrm{w}} / \mathrm{M}_{\mathrm{n}}=1$, was obtained from Polymer Standard Service. All samples were stored and handled in dry nitrogen atmosphere. The polar molecules were dissolved in the apolar solvents at molar concentration of about $X_{M}=0.1$ for $B r E B z$ and at weight fraction $X_{W}=0.1$ for $B z A c$ (determined by mass balance). Dielectric measurements were carried out using a Novocontrol Alpha-Analyzer $\left(v=10^{-2}-10^{7} \mathrm{~Hz}\right)$. The sample cell, made up by a parallel plate capacitor separated by a quartz spacer (geometric capacitance $\sim 90 \mathrm{pF}$ ), was filled by the sample and placed in the nitrogen flow Quatro cryostat for temperature control.

\section{Results and Discussion}

\subsection{BzAc and mixtures of BzAc in IPB or OTP $\left(X_{W}=0.1\right)$}

In Figure 1 we present the isochronal dielectric loss at $1 \mathrm{kHz}, \varepsilon^{\prime \prime}(1 \mathrm{kHz})$, of neat BzAc, 10 wt.\% of BzAc in IPB, and $10 \mathrm{wt} . \%$ of BzAc in OTP. Spectra of the pure weakly polar solvents are also shown. In addition to the $\alpha$-loss peak located near $175 \mathrm{~K}$, the isochronal spectrum of neat BzAc shows a low temperature secondary relaxation $\gamma$-loss peak. As suggested from the molecular structure of BzAc shown in Fig.1, this $\gamma$-relaxation is likely coming from rotation of the $-\mathrm{OOC}-\mathrm{CH}_{3}$ unit at the flexible oxygen bond, which links it to the benzene ring. The intramolecular nature of $\gamma$-relaxation indicates that it is not the JG $\beta$-relaxation [11]. This will be supported by the insensitivity of the $\gamma$-relaxation time to mixing BzAc with OTP and IPB to be discussed shortly below. If the JG $\beta$-relaxation exists in neat BzAc, it must be located at temperatures in between the $\alpha$ and the $\gamma$-relaxation, and hence unresolved.

On mixing $10 \mathrm{wt} . \%$ of BzAc with OTP having much higher $T_{g}$, it can seen from Fig.1 that the $\alpha$-loss peak of BzAc is shifted to about $250 \mathrm{~K}$ while the location of the $\gamma$-loss peak remains practically unchanged. A new secondary $\beta$-relaxation of BzAc now appears in the isochronal spectrum. This must be the JG $\beta$-relaxation of BzAc in the mixture with OTP. It is now resolved because of the JG $\beta$-relaxation of BzAc is further separated from the $\alpha$-relaxation of BzAc in the mixture according to prediction (ii). This process comes from the contribution of dipole fluctuations of BzAc molecules coupled to the motions of the overall system (i.e. mixture OTP/BzAc) in the glassy state. In this sense, the relaxation is truly intermolecular in nature. Its amplitude, frequency and temperature behavior may depend on the characteristics of the apolar solvent but also on those of the dipolar probe, as clearly shown for different halogenated benzenes in ref.[23].

In the mixture of $10 \mathrm{wt} . \%$ of BzAc with IPB, the guest BzAc $\alpha$-loss peak is shifted by approximately 25 degrees to lower temperatures, reflecting the neat host IPB having a lower $T_{g}$ than the neat guest. The location of the $\gamma$-loss peak is practically unchanged. As a result, the $\alpha$ - and the $\gamma$-relaxations of BZAc in the mixture get closer together than in pure BzAc. This together with the prediction (iv) make the JG $\beta$-relaxation of BzAc in the mixture with IPB even harder to resolve than in pure BzAc.

Isothermal loss spectra, $\varepsilon^{\prime \prime}\left(f / f_{\max }\right)$, emphasizing only the contribution from the $\alpha$-relaxation are shown in Fig. 2 for pure BzAc, $10 \mathrm{wt} . \%$ BzAc in mixtures with OTP and with IPB. Several sets of data taken at different temperatures and $f_{\max }$ centered about $1 \mathrm{kHz}$ are shown for the $\alpha$-relaxation of BzAc in $10 \mathrm{wt}$.\% BzAc mixtures with OTP. These isothermal loss data were taken by spectrometer that has a narrow frequency range [23], and the JG $\beta$-relaxation of BzAc found in this mixture at much higher frequencies cannot be displayed together 
with the $\alpha$-relaxation at these temperatures. It can be seen from Fig.2 that its frequency dispersion is much broader than that of pure BzAc, consistent with prediction $(i)$. On the other hand, the frequency dispersion of the BzAc $\alpha$-relaxation in the mixture with IPB is almost the same as that of pure BzAc. Interestingly, the loglog slope of the high frequency flank of the $\alpha$-peak in the mixture is steeper than in the case of pure BzAc. The possible effect of broadening due to the contribution of the secondary relaxation has o be excluded in this case: in fact the data reported in Fig.2 are obtained by using a master curve procedure, i.e. by shifting several spectra obtained at different temperatures and normalizing their intensity and their frequency to that of the loss peak maximum. Therefore, as the different spectra superpose well in the master curve, the effect of the seconday relaxation is negligible, otherwise the shape of the spectra should change with temperature, as the $\alpha$ $\beta$ separation and their mutual contribution change on cooling. Although the concentration of BzAc in the mixture of $10 \mathrm{wt} . \%$ of BzAc with IPB is not large, presence of some residual composition fluctuations and broadening of the $\alpha$-relaxation cannot be excluded. Taking this effect into account and the fact that the $T_{g}$ of IPB is not much lower than that of BzAc, the data seem to support the intrinsic narrowing of the $\alpha$-dispersion of BzAc predicted by (iii).

\subsection{BrEBz and mixtures of $\mathrm{BrEBz}$ in $\mathrm{EB}$ or $3 S\left(X_{M}=0.1\right)$}

The new data of pure bromoethylbenzene $(\mathrm{BrEBz})$, and mixtures of molar fraction $\mathrm{X}_{\mathrm{M}}=0.1$ of $\mathrm{BrEBz}$ in ethylbenzene $(\mathrm{EB})$ and in tri-styrene $(3 \mathrm{~S})$ were taken by broadband spectroscopy. The isochronal dielectric loss spectra of these three samples at $1 \mathrm{kHz}, \varepsilon^{\prime \prime}(1 \mathrm{kHz})$, are presented in Fig.3. In contrast to BzAc, the JG $\beta$ relaxation is already resolved in pure BrEBz both isochronically (see Fig.3) and isothermally (data not shown). In the mixture with $3 \mathrm{~S}$ having a much higher $T_{g}$, the $\mathrm{JG} \beta$-loss peak of $\mathrm{BrEBz}$ becomes more prominent compared with the neat $\mathrm{BrEBz} \alpha$-relaxation and further separated from the $\mathrm{BrEBz} \alpha$-loss peak. This is consistent with prediction (ii). On the other hand, in the mixture with EB having a lower $T_{g}$, the JG $\beta$ loss peak of BrEBz is suppressed, moved closer to the $\alpha$-loss peak, and no longer resolved in the isochronal spectrum, in accordance with prediction $(i v)$.

Isothermal loss spectra, $\varepsilon "(f)$, of pure $\mathrm{BrEBz}$, mixtures of $\mathrm{BrEBz}$ in ethylbenzene $(\mathrm{EB})$ and in tri-styrene $(3 \mathrm{~S})$ $\left(X_{M}=0.1\right)$ all having approximately the same peak frequency are compared in Fig.4. The loss spectrum of pure BrEBz shows once more the resolved JG $\beta$-relaxation (seen as the shoulder in Fig.4, and resolved peaks at lower temperatures). In the mixture with $3 \mathrm{~S}$, the $\mathrm{JG} \beta$-relaxation of $\mathrm{BrEBz}$ at the temperature shown in Fig.4 is located at frequencies much higher than the upper limit of the experimental frequency window. Its presence is assured in isothermal spectra taken at lower temperatures (not shown). It is clear from Fig.4 that the frequency dispersion of the $\alpha$-relaxation of $\mathrm{BrEBz}$ is much broader in the mixture with $3 \mathrm{~S}$ than in the pure state. This is in accord with prediction $(i)$. While in mixture with $\mathrm{EB}$, the $\alpha$-loss peak of BrEBz is narrower than that of pure BrEBz on the high frequency flank, and an excess wing appears instead of the shoulder.

In Fig.4, also the contribution of the fitting function for $\alpha$ - and $\beta$-process is reported for the systems $\mathrm{BrEBz}$ and BrEBz mixed with EB, clearly showing that the $\alpha$-peak is slighly narrower in the pure system than in the lower $\mathrm{T}_{\mathrm{g}}$ mixture. We used for fitting a simple superposition of the two processes, usually performed in literature but arbirtary and strongly questionable, since the two processes are interrelated as shown in ref.[12, $15,16]$. Of course, when two processes are so close each other, no unequivocal procedure of deconvoluton is possible; but it is clear that any estimation of the true broadness of the $\alpha$-peak should result in a narrower (or at least equal) shape than that of the convoluted peaks. Additionally, the contribution of the $\beta$-peak is not vanishing in the mixed system (the relative dielectric strength $\Delta \varepsilon_{\beta} / \Delta \varepsilon_{\alpha}$ is $\sim 0.06$ and 0.04 for the pure and 
mixed system, respectively): the narrowing of the $\alpha$-peak in the mixture of $\mathrm{BrEBz}$ with EB cannot be ascribed to the vanishing of the secondary process.

Again, taking into account of residual spatially heterogeneous broadening by composition fluctuations in the mixture and the fact that the $T_{g}$ of $\mathrm{EB}$ is only $20 \mathrm{~K}$ lower than $\mathrm{BrEBz}$, the intrinsic narrowing of the $\alpha$ relaxation of BrEBz predicted by (iii) accompanied by the decrease in separation between the $\alpha$ - and JG $\beta$ relaxations are consistent with observation.

A comparison of the results of §3.1-3.2 with the literature data for dynamics of guest molecules in host systems with different mobility is interesting. Almost four decades ago pioneering works [25--29] on dielectric spectroscopy of polar guest molecules in apolar hosts (with both lower and higher $\mathrm{T}_{\mathrm{g}}$ than guest systems) gave evidence of pronounced changes of the breadth of the relaxation, mainly interpreted as effect of fluctuation concentration, but also of the emergence of the JG $\beta$-relaxation that was better evidenced [27--28] in mixtures with hosts with higher viscosity. A picture was proposed to explain the dielectric relaxation in solutions of rigid polar molecules in nonpolar solvents, based on the effect of viscoelastic relaxation of the solvent on the reorientation of the guest dipole under the applied electric field [29]: the breadth of distribution of relaxation times in the dielectric loss spectrum would be directly related to the steady-flow viscosity of the solvent, resulting in narrow and broad peaks for solvent with low and high viscosity, respectively. This picture is in agreement with CM preditcions. Recently, the probe rotation dynamics of probe polar molecules in viscous liquids has been studied [30] by dielectric spectroscopy as a function of the the size of the probe molecule relative to the liquid constituents. The change of the breadth of the $\alpha$-relaxation was interpreted in terms of averaging the eniromental fluctuations due to heterogeneous dynamics. Bigger the size, higher is the degree of averaging, and the heterogeneous dynamics seen by the probe is averaged to result in a process with a very small distribution of time up to reach, for the hydrodynamic regime, a Debye-like relaxation. On the other hand, for size of the solute comparable to that of the solvent, a similar dispersion as that shown by the pure solvent is reported. Unfortunately in ref. [30] no experiments with solute of smaller size or with lower $T_{g}$ than the solvent were reported. In these cases, as reported in our experiments, structural relaxation becomes very broad, with a distribution of relaxation time sometime exceeding that of the pure apolar solvent (see for instance $\mathrm{BrEBz}$ in $3 \mathrm{~S}$ (Fig.4), with $\mathrm{FWHM} \approx 2.78$, where for pure 3S FWHM $\approx 1.9$ ). Although the latter results cannot be explain by using the concept of heterogeneous dynamics as presented in [30], they are naturally explained by coupling model. The overall scenario is confirmed by studies of rotational motion of probe molecules in various hosts with different viscosity by time-resolved optical spectroscopy [31], for which the agreement with CM predictions has been tested [32].

\section{Conclusion}

Coupling model predicts the effects on the breadth of the $\alpha$-relaxation and the separation between the $\alpha$ - and JG $\beta$-relaxation times of a guest glass-former by mixing with host glass-former are very different depending on whether the mobility $\left(T_{g}\right)$ of the guest molecules is higher (lower) than that of the host molecules. While the case of $T_{g}$ of the guest mobility being lower than the host had been studied in many systems by experiments and molecular dynamics simulation and the CM predictions tested, studies in the opposite case, when $T_{g}$ of the guest is higher than the host, are quite rare and a comparison with CM predictions was never done. Our experimental data on two such mixtures have shown opposite directions of changes in the breadth of the $\alpha$-relaxation and the separation between the $\alpha$ - and JG $\beta$-relaxation times of the guest glass-former by mixing with host glass-formers having lower and higher $T_{g} \mathrm{~s}$, in agreement with theoretical predictions. 


\section{Acknowledgements}

The work at the Università di Pisa was supported by MIUR-FIRB 2003 D.D.2186 grant RBNE03R78E. MST kindly acknowledges the support from MIUR ("Borse a favore di giovani ricercatori indiani", n. 1451, 28/07/2005"). KLN was supported by the Office of Naval Research and a grant for International Semester

Exchange Collaboration provided by International Materials Institute for New Functionality in Glass.

\section{References}

[1] J.B. Miller, K.J. McGrath, C.M. Roland, C.A. Trask, A.N. Garroway, Macromolecules 23, 4543 (1990).

[2] A. Alegria, J. Colmenero, K.L. Ngai, and C.M. Roland, Macromolecules 27, 4486 (1994).

[3] G.C. Chung, J.A. Kornfield, and S.D. Smith, Macromolecules 27, 5729 (1994).

[4] K.L. Ngai and C.M. Roland, Macromolecules 28, 4033 (1995).

[5] T. Blochowicz, Broadband Dielectric Spectroscopy in Neat and Binary Molecular Glass Formers (Logos Verlag, Berlin, 2003).

[6] S. Capaccioli, K.L. Ngai, J. Phys. Chem. B 109, 9727 (2005).

[7] A. Zetsche and E.W. Fischer, Acta Polym. 45, 168 (1994).

[8] S. Kamath, R.H. Colby, and S.K. Kumar Phys. Rev. E 67, 010801 (2003).

[9] T. Lodge and T.C.B. McLeish, Macromolecules 33, 5278 (2000).

[10] K.L. Ngai, J. Chem. Phys. 109, 6982 (1998).

[11] K. L. Ngai and M. Paluch, J. Chem. Phys. 120, 857 (2004).

[12] R. Böhmer, G. Diezemann, B. Geil, G. Hinze, A. Nowaczyk, M. Winterlich, Phys. Rev. Lett. 97, 135701 (2006).

[13] A. Nowaczyk, B. Geil, G. Hinze, R. Böhmer, Phys. Rev. E 74, 041505 (2006)

[14] S. Capaccioli, D. Prevosto, K. Kessairi, M. Lucchesi, P.A. Rolla, J. Non-Cryst. Solids 353, 3984 (2007).

[15] M. Mierzwa, S. Pawlus, M. Paluch, E. Kaminska, K.L. Ngai, K. L. J. Chem. Phys. 128, 044512 (2008).

[16] K. Kessairi, S. Capaccioli, D. Prevosto, M. Lucchesi, S. Sharifi, P.A. Rolla, J. Phys. Chem. B 112, 4470 (2008).

[17] S. Capaccioli, K.L. Ngai, N. Shinyashiki, J. Phys. Chem. B 111, 8197 (2007).

[18] T. Blochowicz, E.A. Rössler, Phys. Rev. Lett. 92, 225701 (2004).

[19] S. Capaccioli, K. Kessairi, D. Prevosto, M. Lucchesi, K.L. Ngai, J. Non-Cryst. Solids 352, 4643 (2006).

[20] K. Kessairi, S. Capaccioli, D. Prevosto, S. Sharifi, P.A. Rolla, J. Non-Cryst. Solids 353, 4273 (2007).

[21] K. Kessairi, S. Capaccioli, D. Prevosto, M. Lucchesi, P.A. Rolla, J. Chem. Phys. 127, 174502 (2007).

[22] S. Capaccioli, K. Kessairi, D. Prevosto, M. Lucchesi, P.A. Rolla, J. Phys.: Condens. Matt. 19, 205133 (2007).

[23] Md. Shahin and S.S.N. Murthy, J. Chem. Phys. 122, 014507 (2005).

[24] S. S. N. Murthy, J. Sobhanadri, and Gangasharan, J. Chem. Phys. 100, 4601 (1994).

[25] G. Williams and P.H. Hains, Chem. Phys. Lett. 10, 585 (1971).

[26] M.F. Shears and G. Williams, J. Chem. Soc. Faraday Trans. II, 69608 (1973).

[27] G. P. Johari and M. Goldstein, J. Chem. Phys. 53, 2372 (1970);

[28] G. P. Johari and M. Goldstein, J. Chem. Phys 55, 4245 (1971).

[29] G.P. Johari and C.P. Smyth, J. Am. Chem. Soc. 915168 (1969).

[30] W. Huang and R. Richert, Phil. Mag. 87, 371 (2007), and references therein. 
[31] M.T. Cicerone, F.R. Blackburn and M.D. Ediger, J. Chem. Phys. 102, 471 (1995); M.T. Cicerone, F.R. Blackburn and M.D. Ediger, Macromolecules, 28, 8224 (1995); M.T. Cicerone, M.D. Ediger, J. Chem. Phys., 104, 7210 (1996); F.R. Blackburn, C.-Y. Wang, M.D. Ediger, J. Phys. Chem., 100, 18249 (1996). [32] K.L. Ngai, J. Phys. Chem. B, 103, 10684 (1999).

\section{Figure Captions}

Figure 1. Logarithm of dielectric losses at $1 \mathrm{kHz}$ versus temperature for butylacetate: pure (dashed line), mixed with IPB (blue filled stars), mixed with OTP (red filled circles). Also the dielectric losses at $1 \mathrm{kHz}$ of pure IPB (blue open stars) and OTP (red open circles) are shown or comparison. Inset shows the molecular structure of benzylacetate.

Figure 2. Bilogarithmic plot of dielectric losses versus frequency for benzylacetate. Data related to different temperatures have been shifted to obtain master curves, normalizing the intensity and the frequency to that of the loss peak maximum. Continuous line indicates the relaxation peak for pure benzylacetate, as obtained from previously reported fitting parameters [24]. Solid and open symbols indicate data of mixture in OTP and IPB, respectively.

Figure 3. Logarithm of dielectric losses at $1 \mathrm{~Hz}$ versus temperature for BrEBz: pure (open circles) and mixed with EB (full squares) and with $3 \mathrm{~S}$ (full triangles). Data for pure EB (solvent) are shown as open squares. Lines are guides for eyes.

Figure 4. Bilogarithmic plot of dielectric losses, normalized to the peak maximum, versus frequency for pure $\mathrm{BrEBz}$ at $144.15 \mathrm{~K}$ (open circles), for the mixture BrEBz/EB at $125.15 \mathrm{~K}$ (full circles), for the mixture $\mathrm{BrEBz} / 3 \mathrm{~S}$ at $230.15 \mathrm{~K}$ (full stars). Lines are for fitting functions: solid lines represent the overall fits, dashed lines the Havriliak Negami functions for $\alpha$-relaxation and dotted lines the Cole-Cole functions for $\beta$-relaxation. 


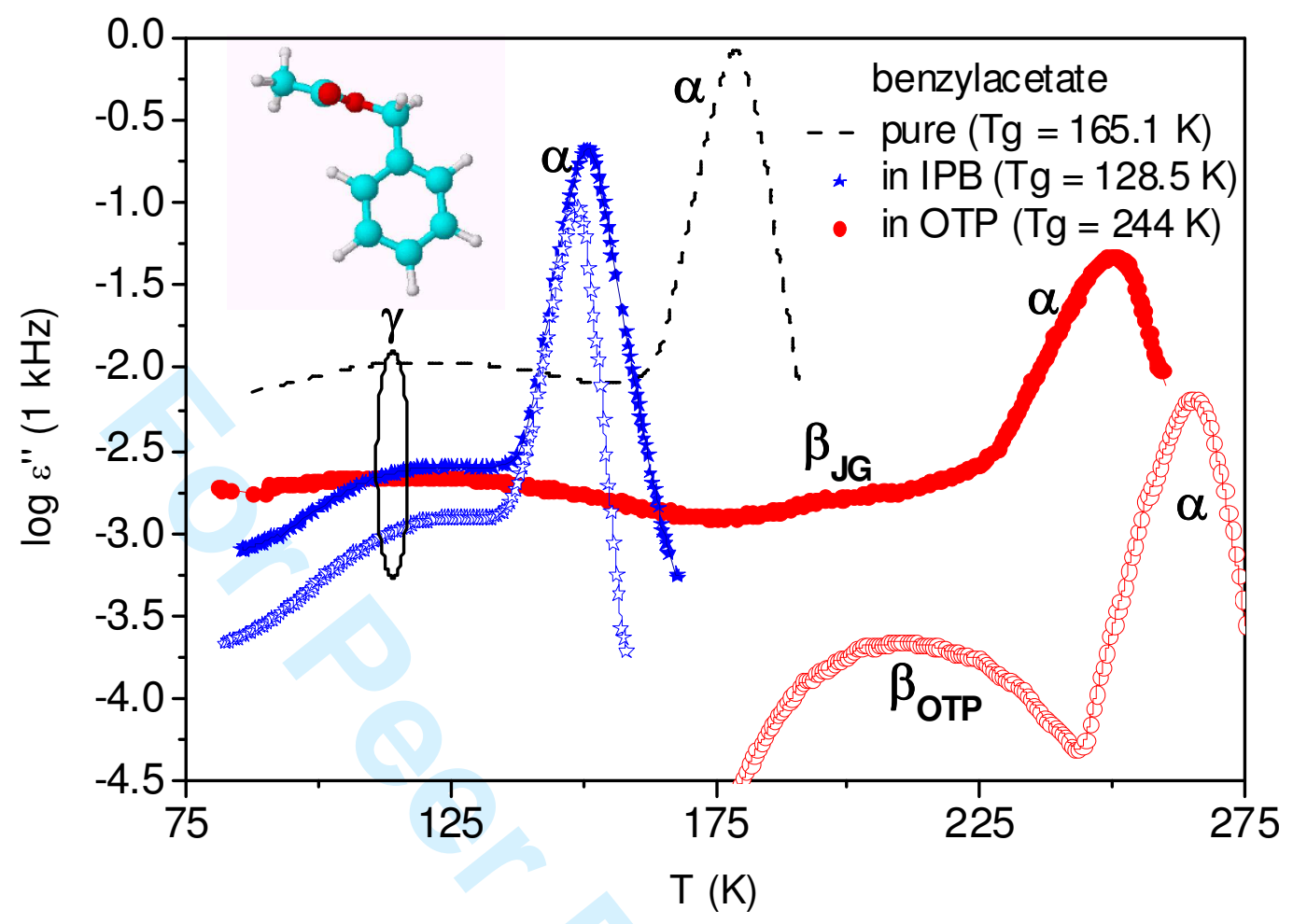

Figure 1

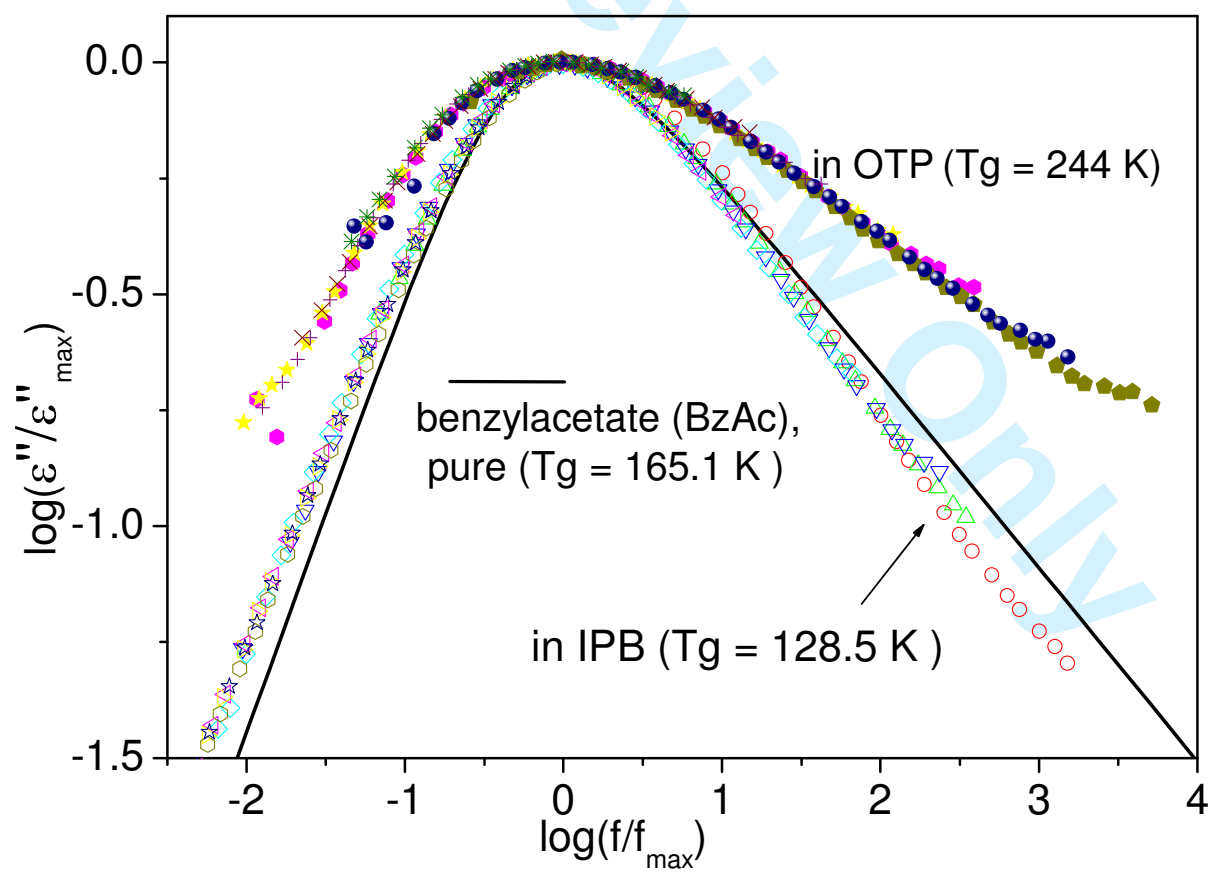

Figure 2 
Figure 3
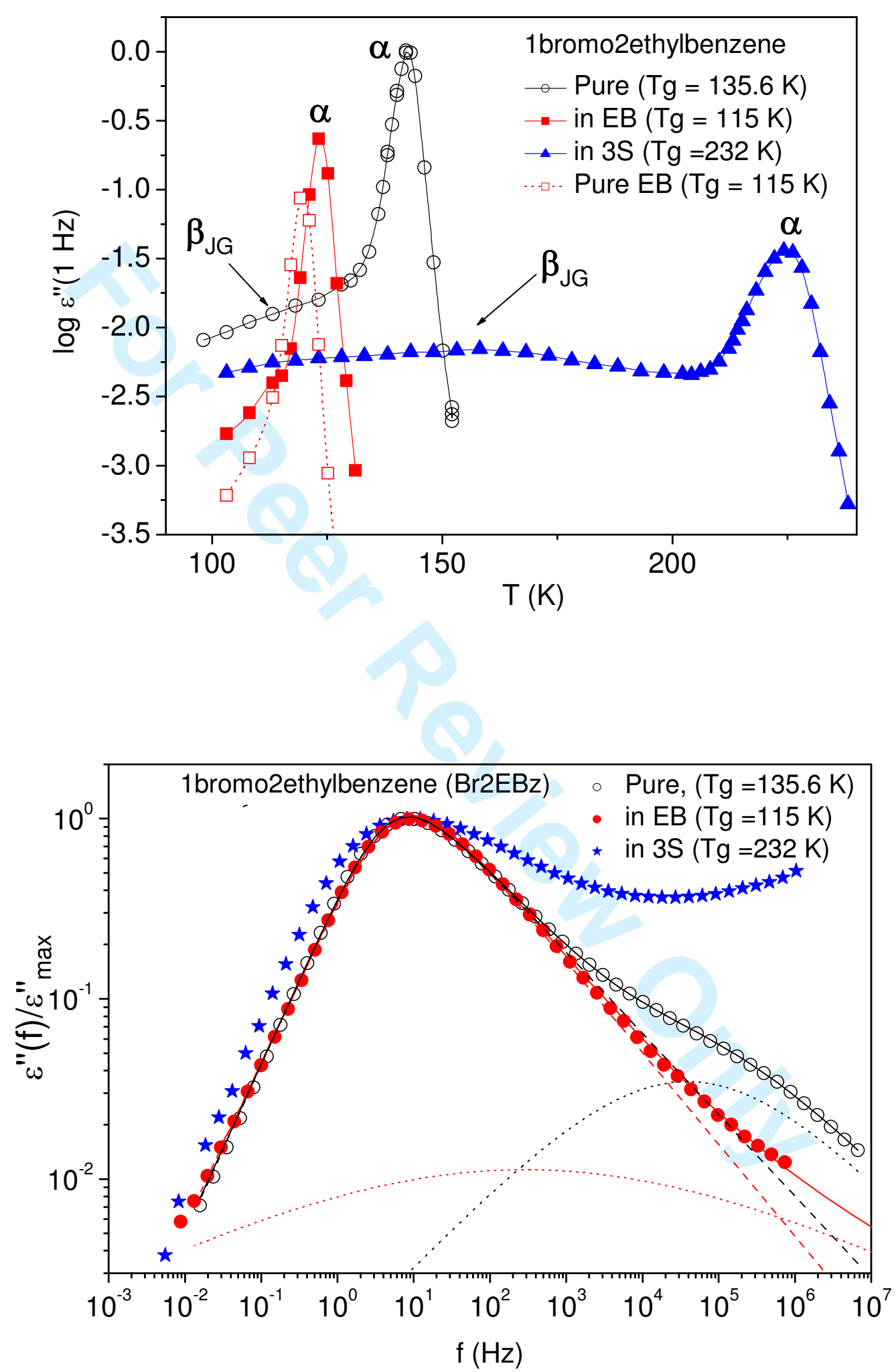

Figure 4 